\title{
Handicap et religion : nouveaux horizons pour la recherche?
}

À propos de :

Schumm Darla, SToltzfus Michael (éds.), Disability in Judaism,

Christianity, and Islam. Sacred Texts, Historical Traditions, and Social Analysis, New York, Palgrave/Macmillan, 2011, 246 p.

Schumm Darla, STolTzfus Michael (éds.), Disability and Religious

Diversity. Cross-Cultural Interreligious Perspectives, New York :

Palgrave/Macmillan, 2011, 234 p.

\section{Lionel Obadia}

\section{(2) OpenEdition}

\section{Édition électronique}

URL : http://journals.openedition.org/assr/25441

DOI : 10.4000/assr.25441

ISSN : $1777-5825$

Éditeur

Éditions de l'EHESS

\section{Édition imprimée}

Date de publication : 30 décembre 2013

Pagination : 103-117

ISSN : 0335-5985

\section{Référence électronique}

Lionel Obadia, « Handicap et religion : nouveaux horizons pour la recherche? », Archives de sciences

sociales des religions [En ligne], 164 | 2013, mis en ligne le 20 février 2017, consulté le 30 avril 2019.

URL : http://journals.openedition.org/assr/25441; DOI : 10.4000/assr.25441

Ce document a été généré automatiquement le 30 avril 2019.

(c) Archives de sciences sociales des religions 


\title{
Handicap et religion : nouveaux horizons pour la recherche?
}

À propos de :

\author{
Schumm Darla, SToltzfus Michael (éds.), Disability in Judaism, \\ Christianity, and Islam. Sacred Texts, Historical Traditions, and Social \\ Analysis, New York, Palgrave/Macmillan, 2011, 246 p.
}

Schumм Darla, StoltzFus Michael (éds.), Disability and Religious Diversity. Cross-Cultural Interreligious Perspectives, New York : Palgrave/Macmillan, 2011, 234 p.

\section{Lionel Obadia}

1 Il y a dans le nord-est du Népal, un village dans lequel réside un vieux tradithérapeute de ma connaissance de la catégorie vaidya et de l'ethnicité Magar, qui a une particularité tout à fait distinctive : en lieu et place de l'un de ses bras, il possède un moignon. Mordu par un serpent lors de travaux des champs dans sa jeunesse, il a dû s'amputer le bras luimême à l'aide de son poignard, un impressionnant kukuri qu'il porte en permanence au ceinturon, pour éviter l'extension du venin à l'ensemble de son corps et une mort certaine, et a lui-même (affirme-t-il) cautérisé la plaie pour éviter l'infection. Ce défaut physique n'a en aucun cas condamné son destin de guérisseur spirituel, bien au contraire : cette mutilation est considérée par les autres villageois de ces hautes vallées himalayennes comme la preuve de l'immense courage du tradithérapeute, mais aussi de son commerce avec le surnaturel. Dans le même village, une chamane dhāmi-jhāânki de l'ethnicité sherpa bénéficiait de la meilleure réputation à trois ou quatre vallées alentours: elle était tout simplement la plus efficace des médiums locaux, et avait développé ses propres techniques de transe, au cours de rituels toujours très spectaculaires, qui avaient contribué à sa renommée. De retour d'Europe après quelques mois, je la retrouve très diminuée par une chute d'arbre (elle est peut-être chamane, elle n'en est pas moins agricultrice, et grimper aux arbres n'a rien d'étonnant) qui a abimé ses 
vertèbres cervicales, et déjà, quelques mois après l'accident, l'une des inattendues séquelles de son invalidité aura été l'étiolement de son pouvoir thérapeutique et de son rayonnement social. Deux figures opposées du handicap, l'un durable et l'autre temporaire, et qui relèvent également d'effets contrastés dans le domaine du surnaturel l'un, par excédent et l'autre, par retrait.

2 Comment le chercheur, et en particulier l'ethnographe, doit-il considérer la présence du handicap dans son champ d'observation social, culturel ou historique, lorsqu'il s'intéresse d'abord au religieux? Une telle question, posée à partir d'une perspective religiologique (d'une approche du monde religieux et non pas d'une approche religieuse du monde) suppose un minimum de symétrie et de s'interroger sur la manière dont le religieux s'invite et est pensé, dans les études sur le handicap.

3 La publication, par l'éditeur Palgrave/MacMillan, de deux volumes (en 2011) essentiellement consacrés aux rapports entre handicap (disability) et religions (monothéismes d'Occident, polythéismes non occidentaux, systèmes de croyances " parallèles ») rappelle à quel point le champ des études sur la religion et celui du handicap ne se sont rencontrés que de manière marginale. L'homo religiosus, tel que décrit dans les sciences religieuses, n'est pourtant pas que cet « homme - blanc - monothéiste occidental - rationnel - riche - en bonne santé » (le chrétien) qui pourtant s'y retrouve de part en part, modèle archétypal ou idéal typique contre lequel s'est longtemps constitué le contre-modèle de l'homme (et encore mieux, la femme) « à la peau noire/jaune/rouge polythéiste - non occidental - crédule - pauvre - affligé de quantité de tares ", contrefigure donc d'un modèle ethno-centré contre lequel les Postcolonial Studies et les Gender Studies n'ont cessé de s'élever. Si le modèle de l'homo religiosus a gagné en épaisseur et en complexité, pour s'éloigner des schémas simplificateurs qui l'enfermaient dans l'anonymat d'un sujet collectif sans âge ni sexe («le» croyant), il demeure en revanche toujours relativement unifié du point de vue d'un corps toujours considéré comme siège de passions religieuses (mystiques) ou moins sacrées (sexuelles), comme "site» psychoaffectif de l'expérience spirituelle, ou comme enveloppe charnelle sur laquelle s'affichent les loyautés et préférences confessionnelles. Ce corps «normal » est la règle les corps abimés, partiellement fonctionnels sont en revanche doublement marginaux : ils le sont par référence à leur marginalité sociale et la marginalisation théorique dont ils font l'objet dans le champ du religieux.

4 Les théologiens d'expression française reconnaissent d'eux-mêmes le retard pris en matière de gestion religieuse du handicap, qu'il s'agisse de lui assigner des significations autres que celles dérivées de références strictement textuelles, ou de mettre en œuvre des dispositifs sociaux d'accompagnement des handicapés suivant des modalités éthiquement acceptables ou théologiquement convenables. "Oser parler du handicap ", telle est l'initiative récemment lancée par l'Université catholique de Lille (qui s'est traduite scientifiquement par un numéro spécial de la Revue d'éthique et de théologie morale, en 2009). Que les religions s'intéressent à des questions d'actualité, qui touchent à la solidarité sociale envers des catégories exclues ou souffrantes n'a en soi rien d'étonnant et par ailleurs, il est relativement aisé de constater que nombre de groupes missionnaires ou à l'action évangélique faisant valoir des compétences en matière de recouvrement des capacités mentales ou motrices déploient des stratégies correspondantes en ciblant expressément les « handicapés » comme une population réceptive à un message religieux. Dans le même ordre de choses, cela fait déjà de longues décennies que la ville de Lourdes, site réputé pour les guérisons miraculeuses en contexte chrétien, dispose d'un service 
d'accueil des pèlerins handicapés, qui forment une large partie de l'audience des sanctuaires locaux - en particulier la grotte de Massabielle (lieu des apparitions de l'Immaculée Conception). La campagne de levée de fonds du Fonds social juif unifié (FSJU) de 2011, qualifiée d'Appel national pour la tsedaka («charité ») qui s'est affichée dans les transports en commun de Paris, a pris pour emblème un vieil homme atteint de la maladie d'Alzheimer et un adolescent trisomique: deux figures du handicap, l'un, d'acquisition tardive, l'autre, de naissance. Mais deux figures pour une même cause : la réduction du stigmate et de l'isolation qui affectent cette partie, souvent laissée dans l'ombre, des communautés confessionnelles.

5 Dans la plupart des cas, la dimension religieuse du handicap (relevant soit d'une signification religieuse attribuée au handicap, soit de dispositions particulières des handicapés à l'expérience spirituelle) a été traitée par des spécialistes du handicap (et non de la religion) qui opèrent alors un détour par les textes sacrés - comme si tout le sens et l'action potentiels se trouvaient concentrés dans le scripturalisme (chez Poizat, 2005 et Segond, 2010). En retour, peu d'initiatives émanent du côté des sciences religieuses d'expression française, tout du moins, en tant que thématique ramassée permettant de constituer un champ de connaissance hybride, car pluridisciplinaire, mais solidement amarré à son objet. Ce n'est actuellement plus le cas ailleurs.

Si la question du handicap (physique ou mental) est traitée de manière dispersée dans les sciences religieuses, elle n'en est pas pour autant absente ou occultée. Le handicap n'apparaît ainsi jamais de manière très directe : ni sur le plan du matériau textuel, qui existe et dans lequel il convenait de puiser les références théologiques pour en dégager les représentations de la déficience et du handicap, ni sur celui des expressions esthétiques du handicap, telles qu'elles sont par exemple données dans la littérature antique (la mythologie antique est peuplée de personnages qui présentent des handicaps physiques, mais sont des héros ou demi-dieux : đedipe est aveugle, Héphaïstos boiteux, etc.) et dans l'art à thème religieux, qui regorge de références en la matière - en particulier dans l'œuvre d'un Bruegel ou de Bosch. Le tableau Les mendiants de Pieter Bruegel l'Ancien (1568) montre des " estropiés » et "gueux » à même le sol, dans ce qui est probablement le jardin d'un hospice: la sémiotique et l'esthétique du handicap expriment plus ou moins directement le rapport du handicap à la société "civile " (exclusion) et aux institutions religieuses (assistance). Avec la sécularisation, les déterminismes symboliques et la pression sociale entourant la figure du handicapé ont changé de registre : la fin des accusations en sorcellerie ou en « diablerie » des boiteux, fous et bossus - en particulier les femmes - a entraîné une réinterprétation et une prise en charge essentiellement médicale de leurs maux. Une laïcisation progressive des institutions communautaires qui a, en moins de deux siècles, progressivement mais sûrement marginalisé les registres religieux des significations et usages entourant le handicap, jusqu’à leur réapparition récente, au cœur des sociétés sécularisées.

\section{De la marge vers le cœur : politiques du handicap et émergence des Disability Studies}

7 Dernièrement, pourtant, le handicap a donné lieu à une littérature d'autant plus importante que la recherche accompagnait, dans les années 1990 et 2000, un certain nombre de dispositifs légaux installés en France pour remettre la question des déficiences physiques et mentales au cœur des débats publics - les années 1970, qui avaient vu la 
formulation des premiers textes de loi favorisant l'intégration sociale des handicaps, n'avaient en effet pas entraîné de véritable transformation de leur statut. Depuis deux décennies, et surtout dans les années 2000, les dispositifs légaux et politiques publiques destinés à l'intégration des déficients se sont multipliés. Entre 1975 et 2005, c'est-à-dire entre les deux grandes lois cadres qui ont été élaborées en France à propos du handicap, et qui poursuivaient l'objectif d'intégrer économiquement et socialement les personnes physiquement et mentalement déficientes, ce sont surtout des petites avancées juridiques qui ont contribué à la reconnaissance des "handicapés", frappés d'une persistante marginalité. Elles n'ont néanmoins jamais vraiment radicalement changé le statut juridique et social des handicapés, d'où une législation itérative qui ne cesse de réglementer la place, toujours équivoque, de ceux qui étaient auparavant catégorisés selon des taxinomies différentes - « infirmes », « invalides ", « déficients », etc.

Dans le domaine académique, et non plus politique, depuis la fin des années 1990, les Disability Studies s'érigent comme un nouveau champ de connaissance, dans une perspective pluridisciplinaire qui dépasse les clivages épistémologiques et méthodologiques, pour se concentrer sur «l'objet» handicap (disability). Les Disability Studies entendent explorer les dimensions sociales, politiques, culturelles et psychiques du handicap. Par-delà la technicité inhérente aux problèmes moteurs et psychiques dont souffrent les handicapés, les Disability Studies ont aussi et surtout introduit des questions plus axées sur le psychologique et l'affectif, comme celles relevant du ressenti subjectif du handicap. C'est alors bien plus la narration du handicap en première personne que sa description morphologique ou mécanique qui est mise au centre de l'analyse, dans un contexte où les déficiences physiques et/ou mentales avaient généralement été enfermées dans des taxinomies et des catégorisations collectives et fondées sur une caractérisation symptomatique objectivée - celles de l'ordre médical. L'écart avec la France est, de ce point de vue, saisissant. Les recherches sur le handicap, développées dans une orientation culturelle avec Charles Gardou (2010), sociologique chez Alain Blanc (2012), historique telle que développée par Stiker (2005), couvrent largement le champ, mais ne donnent pas toujours à la dimension religieuse du handicap toute la surface qu'on lui accorde par ailleurs, de manière motivée ou non ( $c$. infra - la conclusion de cet article).

9 Il a donc manqué, selon toute vraisemblance, que l'intérêt social et scientifique pour le religieux, qui s'est rapidement accru depuis quelques décennies, rencontre celui pour le handicap. La publication des deux volumes dirigés par Schumm et Stoltzfus participe de cette volonté, dans le monde anglo-saxon, d'ouvrir à une exploration plus systématique des relations entre religion et handicap. L'initiative est d'autant plus appréciable que le format de publication - le double volume- permet de sortir du relatif monothéocentrisme dans lequel avaient tendance à s'enfermer les approches religiologiques du handicap. La publication d'un Journal of Religion, Disability \& Health (depuis 1999), qui succède à un Journal of Religion in Disability \& Rehabilitation lequel n'aura eu qu'une brève existence en tant que tel (1994-1998) pallie déjà ce manque : c'est l'une des rares revues exclusivement consacrées aux relations entre le handicap, la santé et la religion, qui n'a évidemment pas d'équivalent en France. La revue Reliance, spécialisée sur le handicap, réserve de temps à autre un article à des thématiques religieuses, mais ici rien de très régulier ne s'est constitué autour de ce croisement de perspectives. 


\section{Le handicap au croisement des monothéismes}

10 C'est dans cette lignée qu'a été publié le premier volume édité par Darla Schumm et Michael Stoltzfus, intitulé Disability in Judaism, Christianity, and Islam, titre au demeurant peu révolutionnaire (mais pouvait-il en être autrement ?) néanmoins sous-titré Sacred Texts, Historical Traditions, and Social Analysis. C'est bien plutôt cette partition qui organise ce premier volume consacré aux traditions historiques, celles qui, par le caractère très englobant et élaboré de leur théologie ou de leur corpus doctrinal, comprennent plus précisément que d'autres traditions (les polythéismes «ethniques» ou locaux) une théorie du corps « sain » et du corps « infirme ». Considérant l'existence d'une littérature scientifique qui a déjà traité la question du handicap dans la perspective d'une tradition particulière, les éditeurs partent $d u$ principe selon lequel les traditions religieuses monothéistes offrent des matrices d'interprétation au handicap, mais ne convergent pas dans une théorie unifiée un phénomène qui, lui-même, déborde perpétuellement les essais de définition. Plutôt que de reprendre encore et toujours les inévitables détours par la normativité des traditions textuelles, il s'avère plus intéressant de souligner la diversité des réponses religieuses au handicap que d'en donner une image monolithique ( Editor's introduction, p. XII-XIII). Il s'agit donc de considérer comment chaque tradition dispose de ressources symboliques particulières et les met ou non en œuvre pour donner du sens au handicap physique ou social, et fournir dans le même temps des schèmes d'action qui inspirent ou encadrent les institutions qui le prennent en charge. Dans cet objectif, une approche par les textes n'est pas inutile en tant qu'elle permet de repérer le champ sémantique du handicap dans différentes religions. Pour Schumm et Stoltzfus, cependant, la référence métaphorique au handicap s'avère déterminante pour toutes les autres formes (praxéologiques et institutionnelles) (id. p. xIX). C'est encore la métaphore qui tisse le fil rouge de ce premier tome, car il révèle toute la complexité de ce que les auteurs appellent "le paradoxe du handicap" (the paradox of disability): les mêmes personnes atteintes de déficiences mentales ou physiques peuvent alternativement ou conjointement être qualifiées de saints ou de miséreux (id., p. xx). S'ensuivent alors une série d'études de cas toutes plus intéressantes les unes que les autres, explorant différentes formes de handicap (surdité, lèpre, vitiligo, cécité, déformations physiques...) dans différentes traditions (judaïsme antique et christianisme primitif, islam traditionnel) plus ou moins inspirées par la tripartition théorique énoncée par Hector Avalos quelques années plus tôt, qui distingue entre « redemptionism » « rejectionism » et « historicism » (le texte sacré comme source garante de la réadaptation, comme figuration du handicap corrigée par les faits - en particulier lorsqu'elle est négative - et enfin le handicap selon le texte sacré, tel qu'il se comprend dans son contexte historique). Les chapitres qui traitent du judaïsme rabbinique (Julia W. Belser et Bonnie L. Gracer) montrent bien combien le handicap est doublement contraint par son écart à l'idéal du prêtre (donc dans une figuration négative), mais par sa tolérance pour les simples pratiquants (donc dans une figuration positive). Celui sur la cécité selon l'épitre de Jean (Jennifer Koosed et Darla Schumm) pointe la fonction heuristique et pédagogique de la métaphore de l'infirmité en contexte religieux. Celui qui aborde la difformité selon Augustin montre une difformité qui change de statut et de valeur selon son régime d'ontologie - terrestre, céleste - (Kristi Upson-Saia). La contribution intellectuelle des autres chapitres, quoiqu'indéniable, n'est pas toujours aussi évidente ni importante - en d'autres termes, l'intérêt des autres chapitres de ce premier volume n'est résolument plus 
dans le domaine de l'exploration scripturaliste des figures du handicap (quel est-il donc? ). La seconde partie s'affirme quant à elle plus clairement orientée vers les aspects sociaux du handicap. Elle s'ouvre sur les difficultés concrètes des musulmans à prier en situation de déficience, et se conclut par une réflexion d'Amos Yong autour de l'amour, de la sagesse, et du handicap comme catégories classiques (pour les deux premières) et authentiquement nouvelle (pour la dernière), de nature à relancer, selon l'auteur, la philosophie de la religion, par la manière dont le handicap amène à réinterroger le sens du mal. Entre les deux, quelques chapitres très intéressants sur la légitimation religieuse de l'eugénisme (Gerald O'Brien et Autumn Molinari), les modalités pratiques de transposition des principes métaphysiques et doctrinaux entourant le handicap à l'action pastorale (Christine James) ou l'action des ONG confessionnelles à destination des sourds dans les pays en voie de développement (Amy Wilson et Kirk Van Gilder). Dans l'économie générale du texte, le volume Disability in Judaism, Christianity, and Islam part, de manière assez attendue, des textes pour s'achever dans les pratiques, et parallèlement, de l'antiquité à la modernité. Mais que faut-il chercher dans les sources religieuses du handicap?

\section{Une question ancienne: sources monothéistes de la charité et régimes d' « infirmités »}

11 Dans le choix de lettres du saint catholique piémontais François de Sales (1567-1622), recueilli à l'occasion des numéros 81 et 82 de la revue l'Anneau D'Or de mai 1958 (dite «revue internationale de spiritualité familiale» lors de son numéro spécial «Saint François de Sales nous parle »), le dévot missionnaire, qui devait évangéliser le Chablais, recommandait en 1604, pour "s'unir à Dieu et au prochain », de "prendre quelquefois la peine de visiter les hôpitaux, consoler les malades, considérer leurs infirmités et attendrir [son] cœur sur celles-ci et prier pour eux en leur faisant quelque assistance » (S'unir à Dieu et au prochain, Annecy, 3 mai 1604, 1958 : 186). C'est le même homme d'Église qui avait placé la charité au cœur des valeurs d'un catholicisme enclin à reconquérir les terres abandonnées au calvinisme et qui rappelait ainsi le rôle central, depuis le Moyen Âge, de l'assistance altruiste à l'endroit de ceux qui sont frappés d'« infirmités » temporaires ou définitives. François de Sales prodiguait aussi des conseils pour la vie de dévotion en soulignant l'impact de ces altérations des facultés sur la religiosité : "quant à la méditation, écrit-il, les médecins ont raison : tandis que vous êtes infirme, il s'en faut sevrer. Et pour réparer ce manquement, il faut que vous fassiez au double des oraisons jaculatoires, et que vous appliquiez le tout à Dieu par un acquiescement entier à son bon plaisir qui ne vous sépare aucunement de lui en vous donnant cet empêchement-là à la méditation... » (Ne cherchez que Dieu, Annecy, 30 mai 1609, 1958 : 211).

Les deux occurrences du terme d'«infirmité » dans une œuvre, de l'une des figures intellectuelles sanctifiées de l'Église catholique, signale, s'il fallait encore s'en convaincre, que l'intérêt théologique du christianisme pour les «infirmités » est ancien, continu et qu'il touche à parts égales les gens du peuple et les membres du corps ecclésiastique. Évidemment, le sens de l'« infirmité » est, en cette sortie de Moyen Âge entrée dans une Renaissance troublée par les guerres de religion, assimilable à celle de «maladie ». Deux significations au moins sont à associer ici à l'idée d'infirmité : une première, strictement profane, d'une déficience temporaire des facultés (à l'occasion d'une maladie, par exemple) qui oblige le prêtre à suspendre son activité, l'autre, plus proche du sacré, cette 
fois, d'une même déficience, mais qui afflige le "peuple » et dans ce cas les causes de l'infirmité sont à rechercher du côté d'un ordre moral qui aurait été transgressé, et l'affliction du côté du châtiment divin ou surnaturel correspondant à la gravité de la faute. La plus explicite référence à l'exclusion du corps (physique et social) de l'infirmité du culte est cette fois énoncée dans le judaïsme, à travers ces célèbres et très cités versets du Lévitique (21:16-23) en ces termes :

"Aucun homme parmi tes descendants, dans toutes les générations, qui serait atteint d'une malformation corporelle ne s'approchera pour offrir l'aliment de son Dieu. En effet, sont exclus du service tous ceux qui ont une infirmité : quelqu'un qui est aveugle ou boiteux, qui est défiguré ou qui a des membres disproportionnés, qui est estropié de la jambe ou du bras, bossu ou nain, affligé d'une taie sur l'œil, qui a la gale, des plaies purulentes ou les testicules écrasés. Aucun descendant du prêtre Aaron ayant une malformation n'offrira à l'Éternel les sacrifices consumés par le feu; du moment qu'il a une malformation en lui, il ne s'approchera pas pour offrir les aliments de son Dieu. Il pourra consommer l'aliment de son Dieu, les offrandes saintes et très saintes, mais il ne s'avancera pas jusqu'au voile et ne s'approchera pas de l'autel, à cause de sa malformation; ainsi il ne profanera pas mes lieux saints, car moi, l'Éternel, je les rends saints. »

La citation, un peu longue, se justifie ici par son caractère de récurrence - il est peu de travaux sur le handicap en religion qui n'en fassent pas mention dans le cadre d'un examen comparatif des conceptions monothéistes (scripturaires) du handicap.

Ce premier régime d'infirmité, inscrit dans le concret et la biologie du corps du prêtre et du fidèle, s'adjoint un second régime, nettement plus métaphorique, cette fois, qui relève de l'évaluation de la loyauté religieuse. C'est ainsi que la Sourate II, dite « la génisse » du Coran réfère explicitement à l'infirmité comme duplicité et distance au divin et à la norme religieuse :

«Il est des hommes qui disent: nous croyons en Dieu et au jour dernier, et cependant ils ne sont pas du nombre des croyants. Ils cherchent à tromper Dieu et ceux qui croient, mais ils ne tromperont qu'eux-mêmes et ils ne le comprennent pas. Une infirmité siège dans leurs cœurs, et Dieu ne fera que l'accroître; un châtiment douloureux leur est réservé, parce qu'ils ont traité les prophètes de menteurs (II : 7-9).»

La référence à l'infirmité est ici clairement de nature métaphorique, pour installer une ligne symbolique entre les croyants et le reste du monde. Mohammed Arkoun, qui a donné du Coran la traduction qui sert ici de source, a réservé en note de bas de page une explication de cette référence : " partout dans le Coran, par les hommes dont le cœur est atteint d'une infirmité, Muhammad entend les hypocrites, les hommes de foi douteuse et chancelante » (Coran, traduction M. Arkoun, Paris: Garnier-Flammarion, 1970, p. 46). Deux premiers régimes à distinguer donc d'un troisième qui situe le handicap au cœur du pouvoir de guérison dont se voient alloués les virtuoses de la religion - prophètes, héros, prêtres... Dans l'Évangile selon Matthieu, Jésus, lorsqu'il pénètre dans le temple, « guérit des aveugles et des boiteux» (21:12/14), un épisode miraculeux parmi bien d'autres. Ce pouvoir spirituel de guérison, qui a nourri une vaste réflexion autour de la part thaumaturgique de l'action religieuse, connaît encore de substantiels développements (comme dans la fonction "pharmakologique » des Théories de la religion de Camille Tarot, 2008). 


\section{Les religions et les handicaps}

16 Si les monothéismes ont déposé l'infirmité dans le cadre de l'ordre et des obligations morales, c'est-à-dire, dans le cadre d'une théologie suffisamment sophistiquée pour pouvoir inscrire le handicap dans une étiologie religieuse, c'est du côté de la "magie » et des cultes « ethniques » que l'on trouve pourtant les plus féconds développements autour d'une anthropologie religieuse du handicap.

Les magiciens, sorciers et autres prêtres des sociétés « tribales » (pour prolonger un usage catégoriel encore en vogue en sociologie) sont plus particulièrement susceptibles d'être des personnes affectées d'une déficience, qui est à l'occasion retournée à l'avantage du "handicapé », désormais chargé d'un pouvoir ou de compétences en matière de commerce avec le surnaturel. Dans leur célèbre «Esquisse d'une théorie générale de la magie » (1902-1903, rééd. 1985), Marcel Mauss et Henri Hubert suggèrent que « N’est pas magicien qui veut : il y a des qualités dont la possession distingue le magicien du commun des hommes. Les unes sont acquises et les autres congénitales; il y en a qu'on leur prête et d'autres qu'ils possèdent effectivement. [...] Sont ainsi destinés à être magiciens certains personnages que signalent à l'attention, à la crainte et à la malveillance publique, des particularités physiques ou une dextérité extraordinaire, comme les ventriloques, les jongleurs et bateleurs; une infirmité suffit, comme pour les bossus, les borgnes, les aveugles, etc. Les sentiments qu'excitent en eux les traitements dont ils sont d'ordinaire l'objet, leurs idées de persécution ou de grandeur, les prédisposent même à s'attribuer des pouvoirs spéciaux » (Mauss \& Hubert, [1903-1904] 1985 : 19-20).

Il existe donc, sous la plume de Mauss et Hubert, une véritable théorie du handicap, qui se libelle comme un codage culturel des écarts au modèle physiquement et psychologiquement «valide » prédominant dans une société, qui rapprochent certains individus plus que d'autres, du surnaturel. Il est notable, dans tous les sens du terme, que cette même théorie, symboliste, en première instance, soit aussi une théorie sociologique du handicap, en tant que catégorisation collective des déficiences: "Remarquons que tous ces individus, infirmes et extatiques, nerveux et forains, forment en réalité des espèces de classes sociales. Ce qui leur donne des vertus magiques, ce n'est pas tant leur caractère physique individuel que l'attitude prise par la société à l'égard de tout leur genre » (id. : 20).

19 Ainsi, la plus grande prudence doit prévaloir lorsqu'il s'agit d'imaginer les rapports entre religion et handicap, dès lors que l'anthropologie est convoquée en tant que discipline, ou que la réflexion se fonde sur du matériau généralement traité par l'ethnographie (les cultures et religions non occidentales). Au plan comparatif de l'anthropologie, et à titre, une fois de plus, de métaphore, Claude Lévi-Strauss a montré que la figure de l'infirme ou de l'estropié ne doit jamais être considérée, dans un corpus mythique, comme une figure personnalisée, mais comme un opérateur théorique des transformations mêmes des éléments du système symbolique : «[...] les mythes confèrent souvent aux infirmes et aux malades une signification positive: ils incarnent les modes de la médiation. Nous imaginons l'infirmité et la maladie comme des privations d'être, donc un mal. Pourtant, si la mort est aussi réelle que la vie et si, par conséquent, il n'existe que de l'être, toutes les conditions, même pathologiques, sont positives à leur façon. Le "moins-être" a le droit d'occuper une place entière dans le système, puisqu'il est l'unique forme de passage concevable entre deux états "pleins" " (Lévi-Strauss, 1964: 61). Ainsi, le double détour 
anthropologique par les cultures et les pensées autres, et le comparatisme transculturel ramènent au jeu subtil de l'exclusion et l'inclusion théorique plus qu'empirique des symbolisations du handicap.

D'une manière alors peu surprenante, le second volume, Disability and Religious Diversity, offre un panorama riche d'études de cas, qui, sur au moins deux plans, s'apparente à une approche anthropologique du handicap: du point de vue méthodologique tous les cas sont empiriques et construits à partir des acteurs rencontrés sur le terrain et donc souvent à partir de conceptions "indigènes »; et les traditions convoquées sortent du cadre habituel des trois grands monothéismes. L'accent est alors mis plutôt sur des approches qualitatives, et en particulier sur des récits à la première personne qui font surgir l'expérience subjective, mais moins en narration égocentrée qu'en tant que contribution à restituer le cadre culturel et social dans lequel le handicap va prendre sens et forme du point de vue religieux. Méthodologiquement féconde, la démarche ne donne néanmoins pas systématiquement lieu aux éclairages attendus, en l'occurrence, la manière dont le vécu subjectif rencontre un répertoire de sens (une théologie) et est réorganisé en conséquence. En outre, l'approche est loin d'épuiser toutes les possibilités de relation entre le handicap et la religion. Il manque d'ailleurs ici de vastes pans à l'analyse : la gestion socioreligieuse du handicap, avec des dispositifs de type «religiosité populaire » (pèlerinage ou séance de thérapie collective), et si le volume insiste sur la manière dont le handicap surgit comme une affliction signifiante du point de vue religieux (pour l'acteur et pour l'institution qui l'encadre), il ne dit rien de la stigmatisation et des pressions sociales, voire religieuses (des religions qui sont ici toujours présentées - ou presque - comme tolérantes et bienveillantes) qui s'exercent sur le handicapé, selon la nature de son altérité.

Les études de cas présentées dans le catholicisme latino (Aime B.Valeras), les mouvements Baha'i (Priscilla Gilman), Wicca (Jo Pearson) ou Native American en Alaska (Lavonna Lovern) demeurent à un niveau d'analyse assez localisé, alors que le chapitre consacré au Mouvement Celtique (Erynn R. Laurie), qui mêle analyse empirique, étude textuelle et contextualisation sociohistorique, est méthodologiquement plus solide que les autres. Une contribution intermédiaire tente de montrer, sans révolutionner le genre, les bénéfices sociaux et moraux que retirent les personnes handicapées lorsqu'elles fréquentent des communautés religieuses (Jeff McNair et Abigail Schindler) avant que ne débute vraiment, mais en ordre relativement dispersé, le comparatisme transculturel que ce second volume voulait mettre en œuvre. Les deux éditeurs (Schumm et Stoltzfus) se réservent deux chapitres, l'un sur le taoïsme et le handicap, plutôt au raz de la tradition textuelle, l'autre sur le bouddhisme et le christianisme, qui s'essaye à relier des récits d'acteurs avec des répertoires religieux - essai intéressant, mais un peu trop court pour être tout à fait innovant. Les conceptions symboliques des Native Americans à propos de la différence (et donc du handicap) se voient réserver un chapitre assez innovant (Lavonna Lovern, encore), mais ce qui fait sans doute la contribution la plus originale de ce second volume est celle (bien trop courte) consacrée comparativement à l'islam, au bouddhisme et au christianisme (Lynne Bejoian, Molly Quinn et Maysaa Bazna) qui montre la nécessité de constituer une méthodologie hybride fondée sur du matériau ethnographique, textuel et sémiotique permettant de saisir le handicap comme une altérité qui convoque, par-delà les différentes traditions religieuses, de mêmes thématiques (justice sociale, agentivité, responsabilité et engagement, p.193) au demeurant très... séculières! 


\section{La variable « religion » : heuristique ou piège à penser?}

Au détour de ce bref panorama et de la lecture de ces deux volumes qui, à n'en pas douter, vont marquer une étape supplémentaire dans la progression (rapide) du champ de réflexion autour de "religion et handicap ", la question doit sans doute (et encore) être (re)posée: en quoi la religion offre-t-elle finalement un éclairage particulier sur le handicap? Et inversement, en quoi le handicap représente-t-il une entrée à l'intelligibilité des croyances et des pratiques religieuses? S'agit-il dès lors d'éclairer le handicap via la religion ou la religion via le handicap ? La dernière question peut, certes, s'avérer un peu attendue et relever de ces slogans réversibles dont fourmillent les sciences humaines et sociales, mais elle mérite d'être posée tant la rencontre des deux champs demeure encore assez fugace et semble reposer sur une persistante asymétrie, selon la perspective adoptée. La tentation de se saisir du religieux sous l'angle des Disability Studies peut apparaître comme une concession à une certaine mode. En se déployant, en effet, les Disability Studies ont tendance, parce qu'elles relèvent de Studies (de champs de connaissance) plutôt que de disciplines, à englober quantité d'objets empiriques ou théoriques pour peu qu'ils soient liés au handicap: culture, société, religion, histoire, et concepts moins généraux (stigmate, domination, reconnaissance, etc.). Mais puisqu'il y a du handicap dans toutes les phases de l'histoire et sous toutes les latitudes, de même qu'à l'évidence le religieux a sans doute imprégné la plupart des sociétés de l'histoire, alors l'un comme l'autre offrent une porte d'entrée particulière à l'intelligibilité du social et du culturel. Mais jusqu'où ces perspectives n'entraînent-elles pas une subordination l'une de l'autre?

Le handicap encourt en effet et pour sa part le risque d'être réduit à une catégorie générale de dysfonctionnement physique ou mental ("le handicap » donc "le handicapé ») ou de n'être étudié que dans le cadre des représentations religieuses qui lui donnent sens dans une matrice théologique bien définie (le handicap selon l'islam, le judaïsme, le christianisme, etc.). Les travaux sur le handicap, pour leur part, peuvent reposer sur une conception assez générale, voire parfois franchement superficielle de la religion et de ses dynamiques : sous la rubrique de "religion", ce sont les bases normatives et textuelles des systèmes officiels de croyance (Segond, 2010) ou une vague sociologie du « retour du religieux » (Poizat, 2005) qui sont mobilisés. Double réduction, donc, qu'il faut pointer du doigt, mais qu'on aurait tort néanmoins de condamner - du point de vue méthodologique, difficile de se passer de la catégorisation et des registres scripturaires d'interprétation ou de généralités sur le contexte environnant. Handicaps et croyances admettent évidemment des significations variables au fil du temps et évidemment il s'agit de rendre à l'analyse la pluralité des contextes, des formes de handicap et les idéologies qui les entourent, d'un côté, et des différents systèmes de croyances et de culte d'un autre. C'est alors un entrelacs complexe de relations entre religion et handicap qui se dégagent et on veut en voir ici au moins quatre : 1) la mise en signification religieuse $\mathrm{du} / \mathrm{des}$ handicap(s) ou la question de l'étiologie symbolique de la déficience chez les handicapés ; 2) la figure des handicapés dans les théologies ou la question de la norme implicite ou explicite de l'ordre (moral, sanitaire, etc.) dans les conceptions religieuses ; 3 ) la place des handicapés dans les activités religieuses ou liturgies - leur intégration ou leur exclusion; 4) le paradoxe d'une reconnaissance religieuse du handicap dès lors que celui-ci devient 
socialement plus saillant, car plus problématique au prisme de processus de sécularisation - ce qui revient, au final, à s'interroger sur la pertinence d'une interprétation religiologique du handicap.

Denis Poizat a, par exemple, proposé de « repenser la question » des rapports du handicap à la religion en explorant la problématique de «l'alliance». On y apprend que les religions ont des textes pour penser le handicap (ce qui n'est pas une nouveauté), que la religion change dans le contexte de la modernité (en est-ce une également ?), que les "dieux fabriquent [aussi] la maladie mentale et la déficience lorsqu'ils punissent les déviants» (Poizat, 2005: 22). Certes, mais il est surtout suggéré que «Les personnes côtoyant le handicap, dans nos sociétés partiellement ou largement laïcisées, sont dans un trouble de l'alliance immanente, unissant les hommes entre eux. Ce trouble de l'alliance est sans doute lié aux vestiges de l'alliance transcendantale, celle des hommes avec leurs dieux" (Poizat, $2005:$ 25). Autrement dit que le problème du handicap est d'être est encore bien trop attaché à l'idée qu'il relève d'un stigmate d'origine surnaturelle : le malaise moderne à l'endroit du handicap prendrait donc racine dans une réminiscence du passé religieux des sociétés sécularisées. Il n'est pas sûr que la sécularisation du handicap, qui va de pair avec celle des sciences sociales de la religion, ne soit pas ainsi religieuse par excès : excès de sens théologique (les traditions religieuses ont-elles toutes pensé le religieux de la même manière, avec le même intérêt ?), de référentialité mythique (combien de dieux estropiés pour des dieux valides selon les corpus et les traditions ?), de signification (l'infirmité est-elle le signe d'un excès ou d'un défaut de sens pour les mythes?), voire de positivité (est-il vrai que l'indigent ou l'estropié se voit systématiquement allouer une place dans un champ symbolique et dans un monde social ?). C'est René Girard objectant à Lévi-Strauss que le handicap dans le mythe n'est pas seulement un élément positif des systèmes de représentation (la carence n'étant, pour Lévi-Strauss, qu'un état intermédiaire entre deux états "pleins»), mais aussi le symptôme d'une victimisation qui n'a rien de symbolique ou qui révèle autre chose, de la violence et de la discrimination, plutôt que de la relation au sein d'un système structuré: «La recherche d'interprétations de plus en plus raffinées ne peut que nous égarer et émousser notre sensibilité aux modalités archaïques de victimisation qui travaillent le soubassement de la mythologie. Encore une fois, faire confiance au bon sens, sans oublier ce que nous savons, nous permettrait de comprendre que les infirmités et invalidités de tant de héros mythiques ne font que les désigner à la foule comme victimes potentielles; ce sont des signes moins ambigus, en fait, que celui de "bouc émissaire" dans les textes de persécution, l'appartenance à une minorité ethnique ou religieuse ", affirme-t-il, dans La voie méconnue du réel (Girard, 2002).

$\mathrm{Si}$, en suivant les sociologies actuelles, on conserve du handicap l'idée qu'il est indissociablement lié à une projection idéologique et à une pression sociale, les tendances générales qui se dégagent autour de lui, le stigmate et son corollaire, la stigmatisation (admirablement exposée par Erwin Goffman il y a quelques années déjà, en 1963), confirment en outre qu'en tant que processus social, l'assignation d'un handicap est théoriquement et souvent empiriquement indépendante du fait religieux - la religion qui, sous la plume du même Goffman, relève du «stigmate tribal ", ou en d'autres termes, un handicap héréditaire. Tout l'effort de Goffman a d'ailleurs été de traduire les concepts de la sociologie religieuse durkheimienne en une sociologie profane (dans sa fameuse théorie des rites d'interaction notamment), alors que Michel Foucault avait, dans une perspective quelque peu différente, mais avec de similaires conclusions, reconstitué la 
marche historique de la folie pour montrer que l'émergence des institutions de contrôle social de la folie est corrélative d'une désolidarisation des troubles mentaux du registre de la possession spirituelle et de la gestion religieuse de la réclusion sociale des déficients (Foucault, 1961).

Il y a donc (au moins) deux facettes du handicap, traitées sous l'angle de la microsociologie et de l'histoire respectivement, qui signalent, s'il fallait s'en convaincre, que, sous bien des aspects, c'est d'abord socialement et culturellement par défaut de religion (ou sous une forme sécularisée), que le handicap se manifeste sous nos latitudes et notre historicité (les sociétés occidentales et dans la modernité). Il émerge aussi partout ailleurs, sous sa forme sécularisée et moderne, dès lors que le système biomédical figure la principale matrice d'interprétation et de gestion sociale des écarts aux normes de validité physique et de «normalité » psychique. Il ne fait toutefois pas de doute non plus que le dit "retour du religieux", dans les mêmes contextes (occidentaux et extraoccidentaux), a entraîné un regain d'intérêt pour les dimensions religieuses du handicap, lequel se trouve alors cette fois requalifié par excès de religion - et donc à la fois désécularisé mais aussi traditionnellement sacralisé : les exemples considérés et présentés dans les ouvrages dirigés par Schumm et Stoltzfus montrent en effet que l'inscription du handicap dans un registre étiologique ou praxéologique de nature religieuse, peut être soit lié à une antique conception théologique (comme dans les monothéismes historiques) qui favorise une inclusion rétrospective, soit à une extension $\mathrm{du}$ sens spirituel d'un groupement plus récent (nouveau mouvement religieux ou mouvance New Age) à des phénomènes qui n'étaient initialement pas ciblés comme les plus significatifs en matière spirituelle.

Cette position particulière, cette duplicité, même, d'un handicap «religionisé » et sécularisé en même temps, interdit de situer la variable "religion» comme plus déterminante qu'une autre dans l'étude des phénomènes psychiques, sociaux ou culturels liés à la déficience. Elle reste foncièrement significative, mais pas nécessairement déterminante pour autant. Conscients de ce risque, les deux ouvrages ici recensés n'offrent ainsi pas une théorie générale de la religion dans le handicap ni du handicap dans la religion. L'ambition est ailleurs. La collection d'études de cas présentés ici vise donc à souligner la diversité des approches, objets empiriques, construits théoriques, démarches méthodologiques déployées pour l'étude d'un phénomène entêtant, complexe et aux approches sinueuses. Les deux ouvrages présentent ainsi l'avantage de situer le champ dans un Work-in-Progress qui avance prudemment dans la voie d'une exploration d'abord cartographique avant que d'être programmatique.

\section{BIBLIOGRAPHIE}

« Oser parler du handicap », Revue d'éthique et de théologie morale, Hors-série, n 256, 2009.

ARKoUn Mohammed (traduction), 1970, Coran (Le), Paris, Garnier-Flammarion.

BLANC Alain, 2012, Sociologie du Handicap, Paris, Armand Colin. 
FouCAult Michel, 1961, L'histoire de la folie à l'âge classique, Paris, Plon.

GARDOU Charles (éd.) 2010, Le handicap au risque des cultures. Variations anthropologiques, Paris, Erès.

GIRARD René, 2002, La Voix méconnue du réel, Paris, Grasset.

GoffMAN Erving, 1974, Les rites d'interaction, Paris, Éditions de Minuit.

-, 1975, Stigmate. Les usages sociaux des handicaps (traduit de l'anglais par Alain Kihm), Paris, Éditions de Minuit.

LÉVI-STRAuss Claude, 1964, Mythologiques. Le cru et le cuit, Paris, Plon.

MAuss Marcel, Hubert Henri, 1902-1903, «Esquisse d'une théorie de la magie ", réédité dans Sociologie et anthropologie, 1950, Paris, Presses universitaires de France, $9^{e}$ édition, 1985, p. 3-144.

Poizat Denis, 2005, «Religions et handicap : le trouble de l'alliance », Reliance, 3 (17) : 19-26.

Schumm Darla, Stoltzfus Michael (éds.), 2011, Disability and Religious Diversity. Cross-Cultural Interreligious Perspectives, New York, Palgrave/Macmillan.

-, 2011, Disability in Judaism, Christianity, and Islam. Sacred Texts, Historical Traditions, and Social Analysis, New York, Palgrave/Macmillan.

SEGOND Lalie, 2010, De la déficience. Représentations, imaginaire, perceptions du handicap dans la littérature contemporaine, Paris, L'Harmattan.

STIKER Henri-Jacques, 2005, Corps infirmes et sociétés, Essai d'anthropologie historique, $3^{\mathrm{e}}$ édition, Paris, Dunod.

TARot Camille, 2008, Le symbolique et le sacré. Théories de la religion, Paris, La Découverte.

\section{AUTEUR}

\section{LIONEL OBADIA}

Université Lyon 2, Lionel.obadia@univ-lyon2.fr 